\title{
Optimization of Composite Material Barrel Based on BP Neural Network Approximate Analysis
}

\author{
Xu Yadong ${ }^{1, a}$, Li Yao ${ }^{1}$ and Chen $\mathrm{Yu}^{1}$ \\ ${ }^{1}$ Nanjing University of Science and Technology, Nanjing 210094, China
}

\begin{abstract}
In order to improve the computational efficiency during the simulation and optimization process for the composite structure, the approximate analysis method was studied. The approximate analysis model was established for the composite material barrel by using BP neural network model. According to the network initialization and training, the model was used to replace the finite element analysis in the optimization process. The results showed that the BP neural network approximate model was effective and reliable, and the optimization efficiency was improved obviously.
\end{abstract}

\section{Introduction}

Composite materials can be designed. In order to design a composite structure with excellent performance in engineering, the structure performance analysis and optimization is needed. However, the actual engineering structure exists the following problems: Firstly, the model should be close to the physical model to obtain more accurate results, therefore, the complexity of the calculation model is high, the calculation has to occupy more computing resources and costs; Secondly, during the optimization design process, the optimization iteration needs to carry on a model analysis and to obtain the global optimal solution every time. That takes a long time to calculate. For example, In the process of adopting genetic algorithm combined with finite element analysis for structure optimization, in order to keep the diversity of population, the large population size is usually needed. The finite element program is needed to calculate each time, which makes the process is very slow.

In order to improve the computational efficiency and to speed up the optimization process, the structure approximate analysis technique was often used to replace the complex model so as to reduce the number of structure reanalysis. The artificial neural network method is a common method in structure analysis, which has a high degree of nonlinear mapping ability. Amounts of literature[1-5] showed that the method had been proved to be effective and reliable in the analysis of engineering structure.

In this paper, according to the optimization design of the composite material barrel of artillery weapon, the approximate model of the structure of the composite material barrel model was established by using BP neural network. And in the optimization process, the approximate analysis was used to replace the finite element analysis to improve the optimization efficiency.

\footnotetext{
${ }^{\mathrm{a}}$ Corresponding author : dillxu@139.com
} 


\section{Composite structure and optimization model}

During the firing process of artillery, the inner wall of the barrel is subjected to high temperature and high pressure gunpowder gas, and the common composite material is difficult to be subjected to this special environment. Therefore, the practical application of composite materials in the pipe is generally with a metal lining. At the same time, considering the actual situation of the processing technology and assembly of the composite barrel, the composite field has some limitations. Fig. 1 is the Schematic of composite material barrel composite position. The composite method is for the front part composite. Fig. 2 is the schematic of a composite part in the barrel. The metal lining is the thickness of the cylinder structure. Before and after the thickness difference of $\mathrm{dh}$, the composite $\mathrm{L}$ is the length of the composite region.R0is the inner diameter of the barrel.h1, h2, h3 and h4are the thickness of the metal lining layer and the thickness of the three layer composite Respectively. The total thickness is the constant value.



Figure 1. Schematic of composite material barrel composite position.

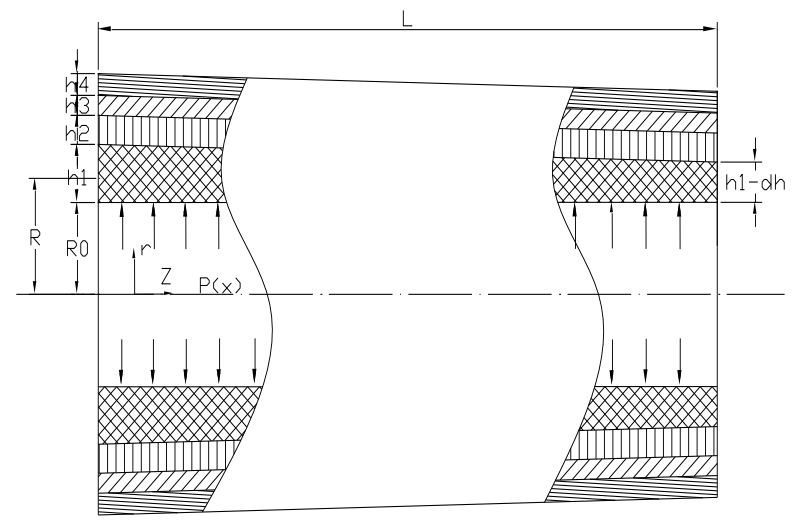

Figure 2. The schematic of the composite part of composite barrel.

According to the above composite structure model, four design variables h1 $\mathrm{h} 2 、 \mathrm{~h} 3$ and $\alpha$ for the composite barrel structure were selected as the optimal design parameters. $\alpha$ is the winding angle of the intermediate layer. The inner layer and outer layer of the composite material winding angle were $90^{\circ}$ and $0^{\circ}$ respectively. The natural frequency of $\mathrm{f}$ was as the target parameter for measuring the stiffness performance. The strength and weight of the barrel were as the constraint conditions. So the mathematical model of the optimization of the stiffness for the composite barrel was as follows. 


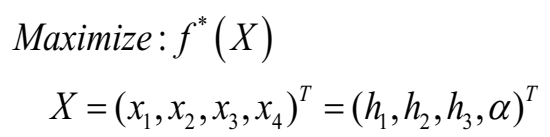

Subject to:

$$
\begin{aligned}
& h_{1}+h_{2}+h_{3}<H \quad\left(h_{1}, h_{2}, h_{3}>0\right), \\
& 0^{\circ} \leq \alpha \leq 90^{\circ}, C_{1}^{*}(X) \geq 1.224, w^{*}(X) \leq \beta w_{0}
\end{aligned}
$$

In Eq.(1), $f^{*}$ is the first order natural frequency of the composite barrel. $C_{1}^{*}$ is the safety factor. $w^{*}$ is the composite barrel quality.w0 is the quality of the whole metal barrel. $\beta$ is the weight loss coefficient. $\mathrm{H}$ is the total wall thickness. $f^{*}, C_{1}^{*}$ and $w^{*}$ are the results respectively obtained by the calculation of the approximate model.

\section{BP neural network approximation analysis model}

\subsection{The network structure and parameters}

BP neural network is the multilayer forward network which uses the error back propagation algorithm to train the weights of the network. The network structure and parameters include: the input / output node of the network, the number of layers, the activation function of each layer and the number of nodes in the hidden layer. In this paper, the BP neural network model was used to simulate the finite element calculation of the structure of the barrel. Its basic network structure was as shown in Fig. 3 .

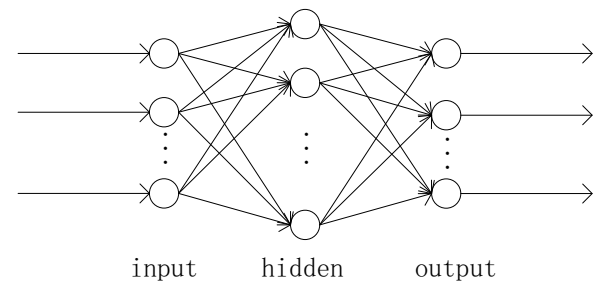

Figure 3. Structure of basic network.

The parameters were set as follows:

(1)Input nodes and output nodes

According to the above analysis, the total number of the composite barrel structure design parameters was four. The performance evaluation index number was three. Therefore, when designing a single multi-input and multi-output network, the input node number is 4 , output node number 3 . When designing a multi-input and single output network, namely the output parameters are designed to a single network individually. The number of output node is 4 . The number of output node is 1 .In order to compare the networks, two kinds of networks were designed, a single output network named BPN-II and a multi-output network named BPN-I. The BPN-I included the strength network, stiffness network and weight network.

(2)The number of plies

The kolmogorov multilayer neural network mapping existence theorem showed that the network concluding a hidden layer could approximate any nonlinear function. For the finite element calculation of the elastic structures, the continuity of the input and output mapping had been proved according to the elastic structure mechanics. Therefore, in order to make the network simple and easy to train, the network of only one hidden layer was selected while satisfying the requirements.

(3) The hidden layer nodes 
The number of nodes in the hidden layer is the main factor that affects the performance of the network. The network approximation ability is weak when the number of nodes is too small. So the training process may not converge. There will appears over-fitting when the number of nodes is too more. The fault tolerance of the network will be lowered. The ability of "generalization" will be reduced. In this paper, by using the network reduction method, the hidden layer nodes of large numbers are set firstly, and then the number was reduced gradually. Ultimately, the 5 nodes were chosen according to the training of the network.

(4) The activation function

The nonlinear approximation capability of BP neural network is the activation function reflected by the tangent type $\mathrm{S}$. The implicit layer selects the $\mathrm{S}$ type activation function generally, while the output layer uses the linear activation function generally.

\subsection{The network initialization and network training}

(1)The network initialization

Before the network training, the initial value of weight and threshold value are set firstly. In order to ensure that the network is not saturated with large weights, the weight of each layer $w$ and threshold $b$ are generally set to be the small random number.

(2) Collection of learning samples

The learning sample collection is essential after determining the structure and parameters of the network. The quantity and quality of the learning sample decide the network generalization ability in a large extent. Generally speaking, learning sample should satisfy compactness, ergodicity and fault tolerance. The learning samples collected by Latin square experimental design method can satisfy these conditions.

(3)The training process of BP network

In numerous training algorithm of BP network training, the Levenberg-Marquardt algorithm has a very fast convergence speed for the small and medium-sized network. The LM algorithm process is shown in Fig. 4. In the training parameters, the expected minimum error was taken as 0.0001 , the maximum number of cycles was 5000, and the learning rate was $0.01 \sim 0.7$.

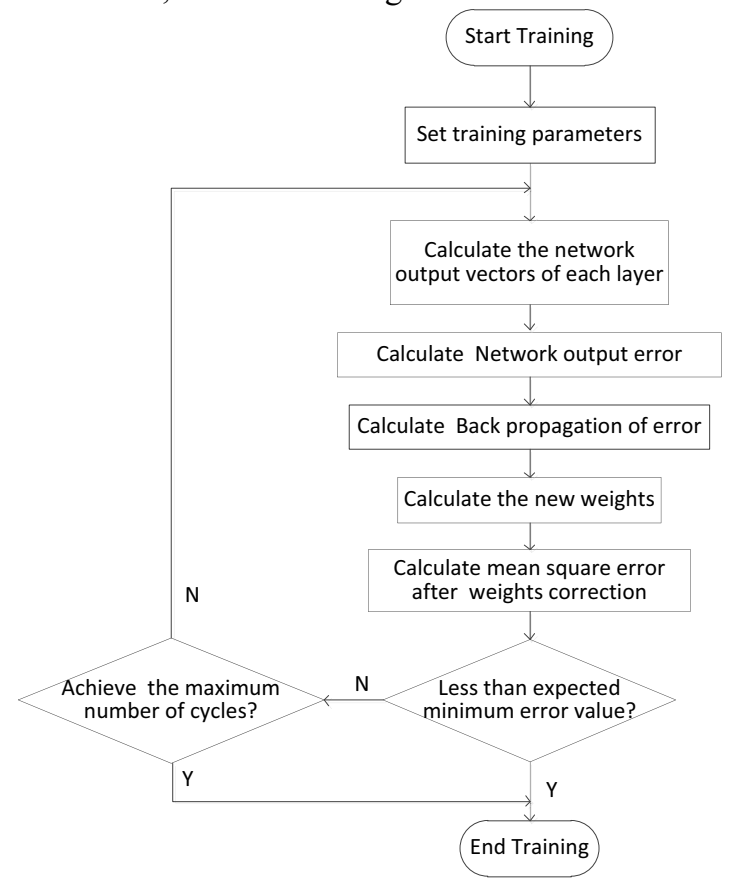

Figure 4. The training process of BP network . 
The above parameters and the network training methods were used to train the $4 \mathrm{BP}$ neural networks which have been established.

\section{The optimal solution and results}

By using BP neural network instead of finite element calculation, the computational efficiency is greatly improved, and the global optimization algorithm is adopted in the combination of approximate model for the optimization of composite structure. Therefore, in this paper, based on the BP neural network (BPN) model, the multi Island genetic algorithm (MIGA) is selected as the main optimization algorithm.

In order to further improve the optimization index after the end of the genetic evolution, combined with the local fast optimization algorithm - Sequential Quadratic Programming (SQP), the formation of the "MIGA - SQP - BPN" comprehensive optimization strategy, The algorithm flow chart is shown in Fig. 5.

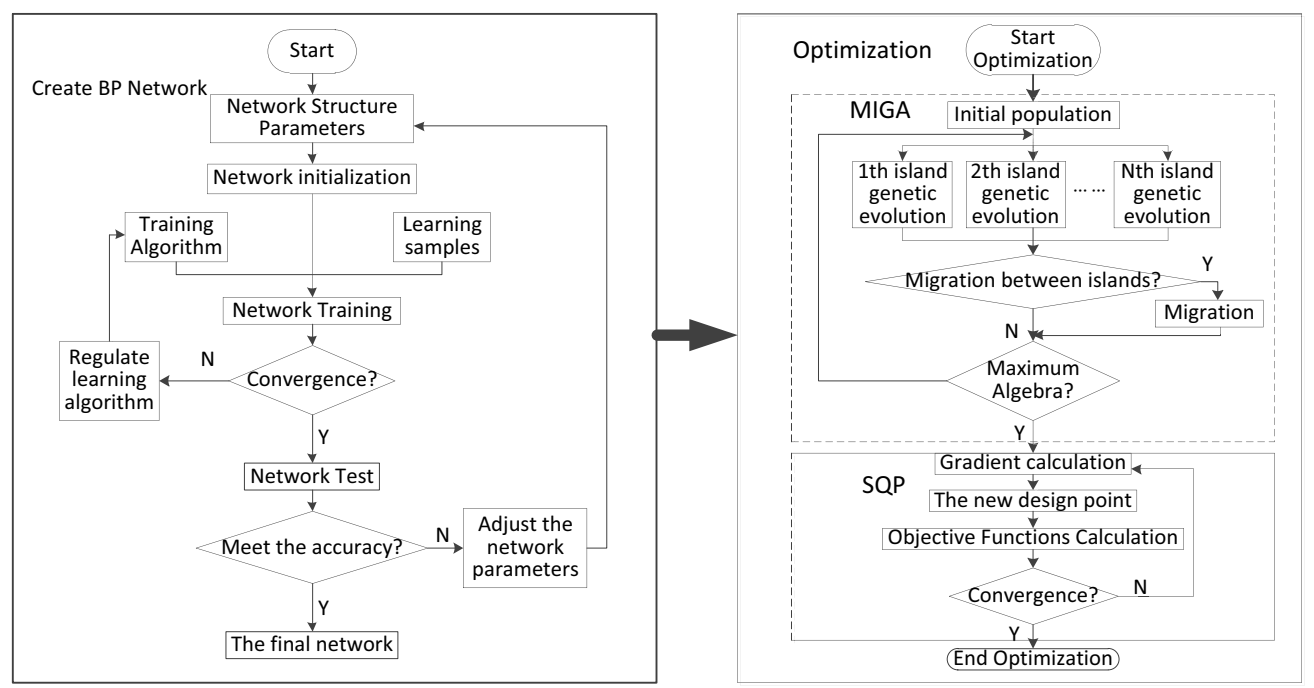

Figure 5. "MIGA - SQP - BPN" comprehensive optimization strategy flow chart.

Multi-island genetic algorithm is a pseudo-parallel genetic algorithm, comparing with traditional genetic algorithm, multi-Island genetic algorithm divides the entire evolutionary population into several subgroups, known as the "island", in each island subpopulations are independent of the selection, crossover and mutation operation regularly, in various islands some randomly selected individuals "migration" operation, will be transferred to the other islands, this way can maintain the diversity of the population, and inhibition of prematurity. Sequential quadratic programming is a mathematical programming method. The basic idea is using Lagrange function for quadratic approximation, generating a sequential quadratic programming sub-problems, sequential quadratic programming by forward difference and the center difference method to calculate the gradient of the objective function. Its main feature is the ability to quickly reach local minima in the local space for given initial solution close. The multi-island genetic algorithm and sequential quadratic programming combining comprehensive optimization, make full use of the complementarity of the two algorithms, multi-Island genetic algorithm to search in the global scope, when close to or reach optimal region, use the sequence quadratic programming to accelerate the local searching speed, making optimization process can quickly reach the optimum design point.

Using "BPN-MIGA-SQP" composite material barrel optimization involves two procedures. First step, creating composite material barrel BP neural network, according to the study sample and the training algorithm to train the network, through the network test, forming network; the second step, 
select genetic parameters, a global genetic evolution by MIGA, SQP local search after the end of the evolution. Finally, the optimal solution is obtained.

According to the optimization model and optimization strategy, the framework of composite material barrel optimization is established, as shown in Fig. 6. The preparation of optimization procedures, integrated BP neural network computing program. To optimize the solution, the MIGA algorithm and the SQP algorithm basic parameters are shown in Table 1.

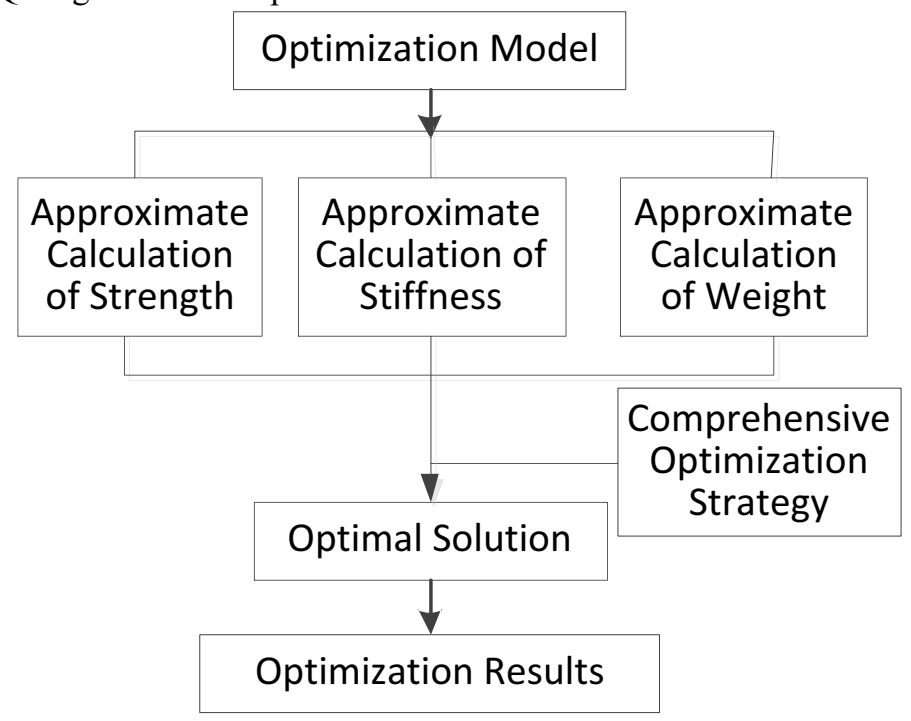

Figure 6. Optimization solving framework.

Table 1. Table of basic parameters of MIGA algorithm and SQP algorithm.

\begin{tabular}{|c|c|c|c|c|c|}
\hline $\begin{array}{c}\text { Max } \\
\text { algebra }\end{array}$ & $\begin{array}{c}\text { Number of } \\
\text { subgroups }\end{array}$ & $\begin{array}{c}\text { Number of } \\
\text { individuals for } \\
\text { subgroups }\end{array}$ & $\begin{array}{c}\text { Crossover } \\
\text { probability }\end{array}$ & $\begin{array}{c}\text { Mutation } \\
\text { probability }\end{array}$ & $\begin{array}{c}\text { Migration } \\
\text { probability }\end{array}$ \\
\hline 200 & 4 & 10 & 0.7 & 0.05 & 0.5 \\
\hline $\begin{array}{c}\text { SQP Maximum number of } \\
\text { iterations }\end{array}$ & \multicolumn{2}{|c|}{ SQP Iteration step } & \multicolumn{2}{|c|}{ SQP Iterative termination } \\
\hline \multicolumn{2}{|c|}{0.001} & 0.00001 \\
\hline
\end{tabular}

Through optimization solution obtained composite material barrel optimal solution. Table 2 gives the comparison of the optimal solution obtained by the approximate model and the optimal solution obtained by the traditional mathematical programming method. In order to show the reliability of the approximate model, the finite element calculation results of the optimal scheme are given in this paper.

Table 2. The design schemes of optimization.

\begin{tabular}{|c|c|c|c|c|c|}
\hline & $h_{1}(\mathrm{~mm})$ & $h_{2}(\mathrm{~mm})$ & $h_{3}(\mathrm{~mm})$ & $\alpha($ degree $)$ & $f_{\max }(\mathrm{Hz})$ \\
\hline Original optimal scheme & 18.33 & 2.02 & 3.28 & 4.07 & 67.80 \\
\hline Latest program & 17.39 & 1.09 & 4.99 & 3.76 & $69.56(69.83)$ \\
\hline
\end{tabular}


By comparison, the optimal solution obtained by "MIGA - SQP - BPN" comprehensive optimization strategy was better than that obtained by the traditional mathematical programming method. The first-order natural frequency than the original optimization scheme improved the $3.36 \%$ and stiffness properties got better; through the comparison of the approximate calculation and finite element calculation of new scheme, the relative error between them was very small, which indicated that the validity and reliability of the BP neural network approximation model of this composite barrel.

\section{5 conclusions}

In this paper, the BP neural network is used to establish the approximate model of the composite barrel structure analysis, and it is applied to the structure optimization of composite material barrel. The results showed that the approximate analysis model based on BP neural network has high approximation accuracy. Due to the adoption of the structure approximation analysis and global optimization algorithm, the optimization efficiency is greatly improved, and the result is more ideal.

\section{References}

1. Cheng Yanxue, Pang Yongjie, Yang Zhuoyi, Research on composite material pressure hulls based on approximation, Ship Engineering, 37(2015)74-78

2. Wang Lei, Lu Jingui, Zhang Jiande, Hua Qi, Numerical empirical study on structural approximation analsysis of neural network for blade of wind turbine, Journal of Vibration, Measurement \& Diagnosis,35(2015)116-119

3. Sei-Ichiro Sakata, Fumihiro Ashida, Masaru Zako, Approximates structural optimization using kriging method and digital modeling technique considering noise in sampling data, Computers \& Structures, 86(2008)1477-1485

4. Olaf Amdt, Thomas Barth, Bemd Freisleben, Manfred Grauer, Approximating a finite element model by neural network prediction for facility optimization in groundwater engineering, European Journal of Operational Research, 166(2005)769-781

5. Olaf Amdt, Thomas Barth, Bemd Freisleben, Manfred Grauer, Approximating a finite element model by neural network prediction for facility optimization in groundwater engineering, European Journal of Operational Research, 166(2005)769-781 